\title{
Proteomics-based signature for human benign prostate hyperplasia and prostate adenocarcinoma
}

\author{
AYODELE A. ALAIYA ${ }^{1}$, MAI AL-MOHANNA ${ }^{1 *}$, MUHAMMAD ASLAM $^{2 *}$, ZAKIA SHINWARI $^{1}$, \\ LAYLA AL-MANSOURI ${ }^{1}$, MAHA AL-RODAYAN ${ }^{1}$, MAHA AL-EID $^{1}$, IRFAN AHMAD $^{2}$, \\ KAMAL HANASH $^{2 \dagger}$, ASMA TULBAH $^{3}$, ALI BIN MAHFOOZ ${ }^{2}$ and CHAKER ADRA ${ }^{1,4}$ \\ ${ }^{1}$ Proteomics Unit, Stem Cell Therapy Program, Departments of ${ }^{2}$ Urology and ${ }^{3}$ Pathology \\ and Laboratory Medicine, KFSHRC; ${ }^{4}$ Transplantation Center, Children's Hospital Boston, \\ Brigham and Women's Hospital, Harvard Medical School, Boston, MA, USA
}

Received November 17, 2010; Accepted December 30, 2010

DOI: 10.3892/ijo.2011.937

\begin{abstract}
Prostate adenocarcinoma often presents at a late stage, due to a lack of early clinical symptoms and lack of accurate objective markers. This study aimed to identify and validate proteomics-based biomarkers useful for prostate cancer diagnosis and to establish a marker-panel for prostate cancer and benign prostate hyperplasia (BPH). Global protein expression patterns in fresh tissue specimens from 8 patients with prostate carcinoma and 16 with BPH were analyzed by two-dimensional gel electrophoresis. Differentially expressed proteins were identified by MALDI-TOF mass spectrometry. We compared our results with those of published studies and defined a set of common biomarkers. We identified 22 differentially expressed proteins between $\mathrm{BPH}$ and prostate carcinomas. The up-regulated proteins in cancer compared to BPH included protein disulfide-isomerase, 14-3-3-protein, Enoyl CoA-hydrase, prohibitin and B-tubulin $\beta-2$. Keratin-II, desmin, HSP71, ATP-synthase- $\beta$-chain and creatine kinase$\beta$-chain were down-regulated. Survey of the literature showed that 15 of our 22 identified proteins have been previously reported to differ in their expression levels between BPH and prostate cancer by other laboratories. The expression patterns of these biomarkers could successfully cluster BPH and adeno-
\end{abstract}

Correspondence to: Dr Ayodele A. Alaiya, Proteomics Unit, Stem Cell Therapy Program, King Faisal Specialist Hospital and Research Center, P.O. Box: 3354, Riyadh 11211 (MBC\# 03), Saudi Arabia E-mail: aalaiya@kfshrc.edu.sa

${ }^{*}$ Contributed equally

'Deceased

Abbreviations: 2-DE, two-dimensional electrophoresis; PSA, prostate specific antigen; $\mathrm{PCa}$, prostate carcinoma; $\mathrm{BPH}$, benign prostate hyperplasia; TURP, trans urethral resection of prostate; RP, radical prostatectomy; MALDI-TOF, matrix-assisted laser desorption/ ionization-time of flight

Key words: $\mathrm{BPH}$, prostate cancer, Gleason scores, proteomics, protein expression, biomarkers carcinomas as well as prostate cancer of low and high Gleason scores. This study validates protein-biomarkers that can be useful for accurate diagnosis and prognostic monitoring of prostate adenocarcinoma. Despite varied prevalence of the disease between different ethnic populations (i.e., high in Sweden, low in Saudi Arabia); the biomarkers indicate that BPH and prostate cancers are biologically 'homogeneous' in their protein expression patterns across wide geographical regions.

\section{Introduction}

Prostate cancer accounts for approximately $25 \%$ of all newly diagnosed cancers and is the second leading cause of cancer deaths among the male population in America $(1,2)$. While the prevalence of prostate cancer is relatively low in Saudi Arabia, it is the most commonly diagnosed cancer in men in Sweden (3). Even though the molecular variations in prostate carcinomas across wide geographical regions have not been extensively studied, the disease is relatively more common among AfroAmericans and often present with advanced stage disease compared to what is observed in American white men $(4,5)$.

An improvement and widespread availability in the measurement of PSA, such as PSA density, PSA volume and adjusted age-specific PSA ranges has resulted in early disease diagnosis in the Western world (6). However, many malignant cases still elude early detection and patients often present with metastasis at time of diagnosis $(7,8)$ especially in the less developed world. Patients with occult metastases usually do not benefit from radical prostatectomy or radiotherapy and often respond less favorably to hormonal treatment $(5,9)$. Prostate cancer is biologically heterogeneous with unpredictable aggressive behavior and currently the molecular events underlying its development and progression are poorly understood.

Proteomics studies have reported differences in protein expression profiles between BPH and prostate carcinomas. A number of proteins were identified as potential diagnostic or prognostic markers of prostate cancers (10-12). Among the identified proteins from human prostate cancers is Protein disulfide isomerase, Enoyl CoA-hydrase, Prohibitin, Cytokeratin-18, HSP-60, HSP-71 kDa, glutathione-S-transferase- $\pi$, 
superoxide dismutase, tropomyosin-2 and triose phosphateisomerase $(12,13)$. The pattern of expression of many of these proteins in prostate cancer is similar to that found in breast and ovarian cancer. This suggests a high degree of similarity in the protein expression profiles of different epithelial tumors $(10,11,14)$. The usefulness of the identified proteins as potential prostate cancer markers are yet to be validated in a large cohort of clinical materials within an ethnic group or across different ethnic populations. With the advancements in molecular biology technology, identification of PCA progression may become more accurate and easier at protein level using protein expression patterns unique to each patient.

The aims of this study was to use the proteomics technique to: i) characterize the pattern of polypeptide expression in $\mathrm{BPH}$ and prostate cancer, ii) identify biomarkers useful for diagnosis and iii) establish a marker panel for prostate tumors. Another objective was to compare our results with those of other published studies and to allow us to define and validate a set of common prostate tissue-associated biomarkers across distinct ethnic regions of the world. Furthermore, to determine whether there are ethnic differences in the protein expression patterns of BPH and prostate carcinoma in Sweden and Saudi Arabia.

To our knowledge, this is the first attempt whereby 2-DE fingerprint data were used to evaluate biological variability of BPH and prostate adenocarcinoma samples between different ethnic populations.

\section{Materials and methods}

We analyzed fresh surgical biopsies from $16 \mathrm{BPH}$ patients obtained by Trans Urethral Resection of the Prostate gland (TURP) and eight malignant tumors obtained by radical prostatectomy. The patients had no prior hormonal treatment or radiotherapy and the patient characteristics are indicated in Table I.

The gross samples for routine pathology and samples for 2-DE were examined by two observers and independently classified as BPH and carcinomas. Routinely 12 core biopsies are taken for histopathological diagnosis of BPH. Furthermore, all BPH samples were histologically confirmed as epithelial predominant and malignant samples as adenocarcinoma. All patients were recruited at the Department of Urology, KFSH\&RC following written and signed informed consent. The Office of Research Affairs of the KFSHRC approved the study.

Sample preparation protocols. We earlier reported that the quality and reproducibility of 2-DE gels largely depends on adequate sample preparation procedures $(15,16)$. The qualities of samples obtained by TURP were comparable with radically resected tissue samples and were judged as representative as previously described (15). Briefly, sample representativity was assessed by cytology and histological evaluation and only samples with $>80 \%$ tumor cells were included in the study. Following 2-DE separation, low quality sample images judged by markedly low number of total resolved gel spots as well as total optical density were excluded from the analysis as previously described (17). Tumor tissues were homogenized in ice-cold RPMI-1640 medium and the preparation of cells for 2-D gel analysis was performed as previously described (10).
Protein separation: two-dimensional gel electrophoresis $(2 D E)$ protein array. High-resolution two-dimensional gel electrophoresis (2-DE) was used for protein separation. 2-D electrophoresis was performed, using precast immobilized $\mathrm{pH}$ gradient (IPG) strips (pH 4.0-7.0, linear; Bio-Rad) in the first dimension (IEF). Samples were applied via in gel rehydration of $17 \mathrm{~cm}$ IPG strips overnight in sample solution diluted to a total volume of $350 \mathrm{ml}$ with $8 \mathrm{M}$ urea, 2\% CHAPS, 2\% IPG buffer (pH 4.0-7.0, linear), 0.3\% DTT and a trace of bromophenol blue. For analytical runs, $150 \mu \mathrm{g}$ proteins were loaded on each IPG strip and focusing was carried out for $59500 \mathrm{Vh}$ using six steps of combined ramping of gradient with step and holds constant voltage. After IEF separation, the strips were immediately equilibrated twice over $15 \mathrm{~min}$ with $50 \mathrm{mM}$ Tris- $\mathrm{HCl}, \mathrm{pH}$ 6.8, $6 \mathrm{M}$ urea, 30\% glycerol and 2\% SDS. In the first equilibration solution, DTT (2\%) was included, and $2.5 \%$ w/v Iodoacetamide was added in the second equilibration step to alkylate thiols. SDS/PAGE was performed using $12 \% \mathrm{SDS} /$ polyacrylamide homogeneous large gel format $(25 \times 20 \mathrm{~cm}$ x $1.5 \mathrm{~mm})$. The strips were held in place with $0.5 \%$ agarose dissolved in SDS/Tris running buffer and electrophoresis was carried out at constant 100 voltage and temperature $\left(10^{\circ} \mathrm{C}\right)$ overnight until the tracking-dye reached the bottom of the gel. After electrophoresis, gels were stained with silver nitrate solution.

Biological and analytical sample replicates. Histological and cytological evaluations of all samples were done to ensure sample representativity. Two randomly chosen biological samples; run on two duplicate gels resulting in four analytical replicates were used to assess method reproducibility. The gel electrophoresis of the two samples was independently run on two different occasions. The reproducibility was then measured by the average correlation coefficient of the total optical densities between the pairs of gels. Because of limited quantity in amount of protein harvests from clinical samples and combined with a satisfactory average correlation coefficient value of the biological replicate analysis; all biological samples were run on single analytical gel.

Image and data analysis. The silver-stained 2-D gels were scanned at $100 \mu \mathrm{m}$ resolution (12 bits/pixel) using a GS 800 calibrated laser densitometer and data were analyzed using the PDQUEST ${ }^{\mathrm{TM}}$ software v 8.1 (Bio-Rad). For the group sample comparison a 'standard' or 'master gel' was created from one of the samples. The gel with the best resolution and with the highest number of resolved spots was chosen to be the reference gel to link the rest sample gels for differential expression analysis. Polypeptide quantity was expressed as parts per million (ppm) of the total integrated optical density.

Data preprocessing/data analysis. We used a difference of $\geq 2$-fold change as a threshold for marked quantitative difference between sample pairs. Additionally, significantly differentially expressed protein spots were selected using two different statistical methods (Student's t-test and Partial Least Square analysis, features available in PDQuest 2-DE analysis software program). The generated datasets from PDQUEST were normalized prior to multivariate analysis and the data were subjected to hierarchical clustering analysis using the J Express 
Table I. Clinical and pathological characteristics of the samples analyzed.

\begin{tabular}{|c|c|c|c|c|c|c|}
\hline No. & Sample & Age & Diagnosis & Staging & Gleason $^{a}$ & $\mathrm{Rx}$ \\
\hline 1 & UP-02 & 76 & $\mathrm{BPH}$ & - & & TURP \\
\hline 2 & UP-04 & 72 & ADC prostate & T3aNOM0 & $7(3+4)$ & Radical prostatectomy \\
\hline 3 & UP-05 & 75 & $\mathrm{BPH}$ & - & - & TURP \\
\hline 4 & UP-06 & 65 & $\mathrm{BPH}$ & - & - & TURP \\
\hline 5 & UP-07 & 74 & $\mathrm{BPH}$ & - & & TURP \\
\hline 6 & UP-08 & 73 & $\mathrm{BPH}$ & - & - & TURP \\
\hline 7 & UP-09 & 71 & ADC prostate & T2N0M0 & $5(2+3)$ & Radical prostatectomy \\
\hline 8 & UP-11 & 65 & $\mathrm{BPH}$ & - & - & TURP \\
\hline 9 & UP-12 & 62 & $\mathrm{BPH}$ & - & - & TURP \\
\hline 10 & UP-13 & 77 & $\mathrm{BPH}$ & - & TURP & TURP \\
\hline 11 & UP-15 & 71 & $\mathrm{BPH}$ & - & - & TURP \\
\hline 12 & UP-16 & 55 & Ca prostate & T2N0M0 & $7(3+4)$ & Radical radiotherapy + channel TURP \\
\hline 13 & UP-17 & 85 & $\mathrm{BPH}$ & & - & TURP \\
\hline 14 & UP-18 & 64 & ADC prostate & T2N0M0 & $7(3+4)$ & Retropubic prostatectomy \\
\hline 15 & UP-19 & 62 & $\mathrm{BPH}$ & - & & TURP \\
\hline 16 & UP-20 & 75 & $\mathrm{BPH}$ & - & - & TURP \\
\hline 17 & UP-22 & 80 & $\mathrm{BPH}$ & - & - & TURP \\
\hline 18 & UP-23 & 58 & ADC prostate & T3cNOMO & $7(4+3)$ & Retropubic prostatectomy \\
\hline 19 & UP-24 & 63 & $\mathrm{BPH}$ & - & & TURP \\
\hline 20 & UP-25 & 82 & $\mathrm{BPH}$ & - & - & TURP \\
\hline 21 & UP-27 & 80 & BPH & - & - & TURP \\
\hline 22 & UP-28 & 73 & ADC prostate & T2NOM0 & $5(2+3)$ & Radical prostatectomy \\
\hline 23 & UP-33 & 66 & Ca prostate & T3aNOM0 & $5(2+3)$ & Radical prostatectomy \\
\hline 24 & UP-34 & 72 & Ca prostate & T3aNOM0 & $5(2+3)$ & Radical prostatectomy \\
\hline
\end{tabular}

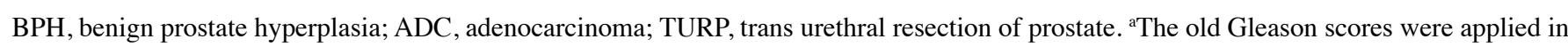
this study as against the modified Gleason system reported after the Gleason consensus conference in 2005 (22).

Pro V1.1 software (java.sun.com) as previously described $(18,19)$.

Protein identification by in gel-digestion and MALDI-TOF-MS analysis. Approximately 500-750 $\mu \mathrm{g}$ protein was loaded for micro preparative runs for peptide mass fingerprinting (PMF). The observed differentially expressed protein spots on 2D gels were selected and systematically cut/picked from Instant Blue-stained gels (Expedeon ${ }^{\mathrm{TM}}$ ) by robotic Proteome Works Plus Spot Cutter (Bio-Rad, Hercules, CA). The gel plugs were deposited into a 96-well micro-titer plate with $\sim 1-5$ gel pieces per well for further in-gel protein digestion. Automated digestion was performed essentially as described in the old Mass PREP Station Digestion protocol 5.7S (Micro Mass UK) with minor modifications. The gel plugs were washed with destaining solution (100 mM Ammonium bicarbonate in water) at $37^{\circ} \mathrm{C}$. After two destaining steps, the buffer was removed, and the gel plugs were dehydrated by adding acetonitrile. Reduction and alkylation steps were done using freshly prepared DTT and Iodoacetamide respectively. Sequencing grade porcine trypsin (Promega) solution $(20 \mu \mathrm{g} / \mathrm{ml}$ stock, diluted to $0.0375 \mathrm{mg} / \mathrm{ml}$ in $25 \mathrm{mM} \mathrm{NH}_{4} \mathrm{HCO}_{3}$ buffer) was then added to restore the gel plug to its original volume. Digestion was carried out at $37^{\circ} \mathrm{C}$ for $4.5 \mathrm{~h}$. Resulting peptides in the gel plugs were extracted once using formic acid. The extracted peptides $(0.8 \mu \mathrm{l})$ were spotted with $(0.8 \mu \mathrm{l})$-cyano-4-hydroxycinnamic acid matrix, $10 \mathrm{mg} / \mathrm{ml}(1 / 1 \mathrm{v} / \mathrm{v}$ ACN/0.1\% aqueous TFA) onto MALDI Target plate. The whole process was performed using the Janus $^{\mathrm{TM}}$ Automated Mass Prep Station (Perkin-Elmer). Peptide mass fingerprinting MS spectra were acquired on a bench top MALDT-TOF Micro Mx (Waters, Manchester, UK). Each spot was analyzed in positive ion reflector mode, by accumulating signal with up to 1000 laser shots (20 sub-spectra of 50 shots) over the $\mathrm{m} / \mathrm{z}$ range of 800-3000 Da. ACTH (Sigma, St. Louis, $\mathrm{MO}) ;[\mathrm{MH}]+=2465.199 \mathrm{Da}$ was used for lock mass correction for every acquisition at a concentration of $500 \mathrm{fmol}$ spotted on target with-cyano-4-hydroxycinnamic acid as matrix. A standard digest of Enolase or alcohol dehydrogenase (ADH) at concentration of 250-500 fmol was spotted on target as multipoint external calibrant. Mass Lynx Global Mass-Informatics v4.0 (Waters) was used for all automated data acquisition. The acquired MS data were background subtracted, smoothed and de-isotoped at medium threshold. Protein Lynx Global Server (PLGS) 2.2 (Waters) was used for all automated data processing 


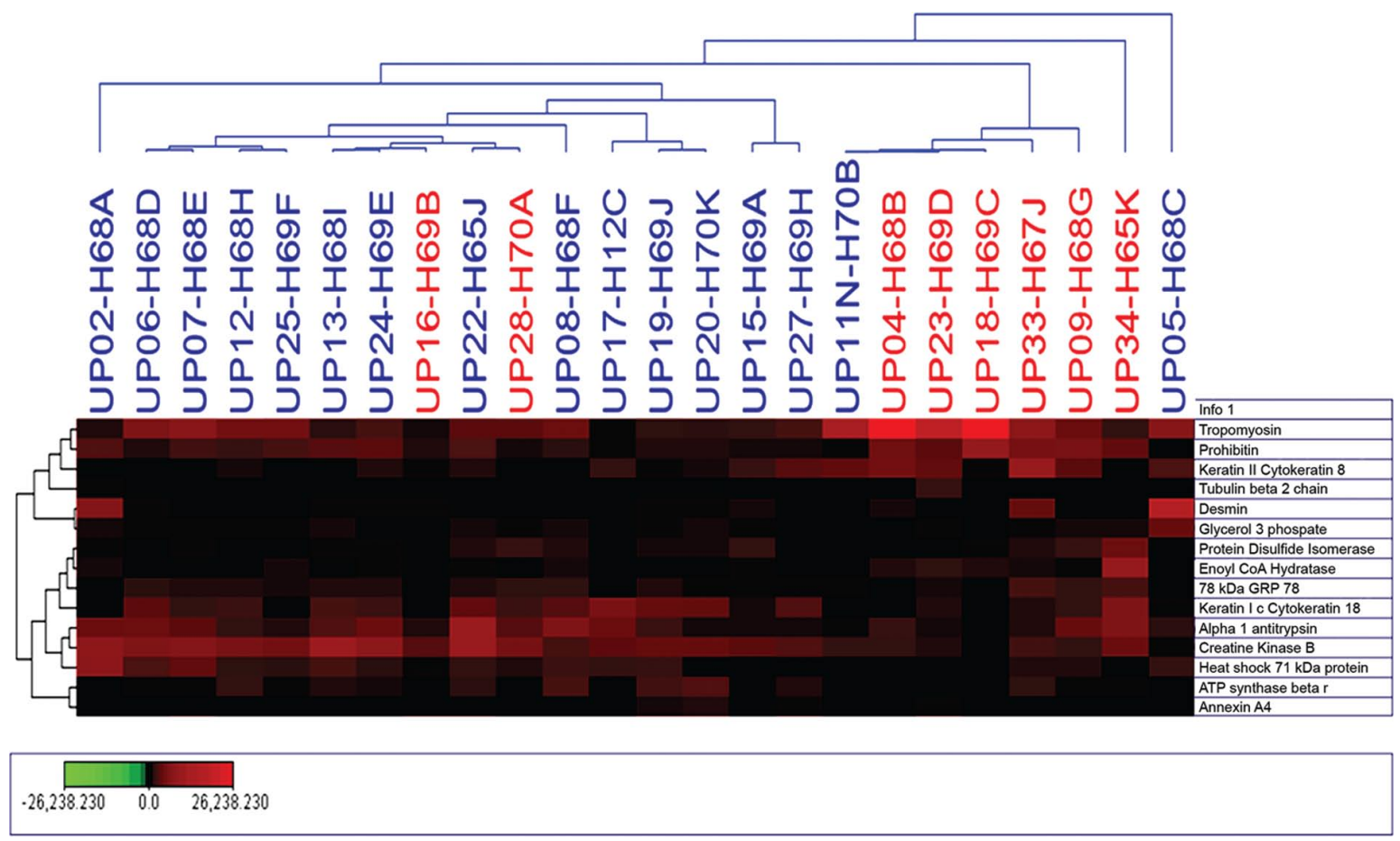

Figure 1. Hierarchical Cluster analysis using the expression patterns of 15 proteins that have been identified both in this study and previously published studies between benign prostate hyperplasia (blue) and prostate carcinoma (red). The dendrogram was generated using the Pearson Correlation distance metric and an average linkage clustering method from the J-Express software.

and database searching. The generated peptide masses were searched against a protein sequence database (Swiss-Prot) using the PLGS 2.2 for protein identification (Waters).

The databank search query parameters includes taxonomy as Homo sapiens, peptide tolerance of $100 \mathrm{ppm}$, estimated calibration error of $0.025 \mathrm{Da}$, molecular weight/pI range of 0-200000 Da/0-14, three (3) minimum peptides to match, trypsin as primary digest reagent, 1 missed cleavages, fixed modifications-carbamidomethyl cysteine and variable modifications of oxidation methionine. A MASCOT protein scores for combined MS spectra of $>60$ were considered statistically significant $(\mathrm{p}<0.05)$. In addition to other parameters, (predicted/observed $\mathrm{pI} / \mathrm{Mr}$, probability, scores etc.) for confirmation of identified proteins after database search, the digested peptides are analyzed 2-3 times for PMF on different MALDI plates and at different runs. Only protein identifications that give the same result in all the runs for each spots are considered as unambiguous protein identification.

\section{Results}

Protein expression in benign and malignant tumor tissues. We have characterized the expression of multiple proteins in human prostate carcinoma and in BPH using the technique of 2-DE. Tumor cells were collected from 16 patients diagnosed with BPH and eight prostate carcinoma patients (Table I).

The method reproducibility was evaluated by running two separate samples more than once and showed an average correlation coefficient of 0.88 as previously described for breast, ovarian and prostate tumors $(12,15,16,20)$. Thereafter each sample was, run once and individual sample of the same diagnosis was considered as replicate under a cohort of analysis group (biological replicate for PBH vs. biological replicate for $\mathrm{PCa}$ ). The average expression levels were taken for comparison between two sample groups being analyzed.

An average 1175 spots were resolved on $25 \times 20 \mathrm{~cm} 2-\mathrm{DE}$ large format gels, and $>75 \%$ of the spots were successfully matched among all gels.

We observed statistical changes in the expression of 47 proteins between BPH and prostate carcinomas using Student's t-test analysis $(p<0.05,98 \% C I)$. Only 22 of the 47 protein spots were successfully identified using MALDI-TOF MS analysis. Among the proteins that are highly expressed in prostate cancer than BPH are protein disulfide isomerase, 14-3-3 protein, Enoyl CoA hydrase, prohibitin and Tubulin- $\beta-2$. Proteins that show increased levels of expression in BPH compared to cancer are Keratin II, Desmin, HSP-71 kDa, ATP synthase $\beta$-chain and Creatine kinase $\beta$-chain.

Validation of differentially expressed for tumor classification. Fifteen of the 22 identified proteins were previously been reported to differ in expression between BPH and prostate carcinomas (11-13). We used the expression patterns of these biomarkers for clustering analysis of BPH and prostate adenocarcinoma and almost all samples were correctly classified, as shown in Fig. 1. The locations of some of the identified proteins on the 2-DE gel are shown in Fig. 2 and names and other characteristics of the protein spots are listed in Table II.

From the above result, it is likely that malignant potential, both stage and grade, influences the discriminating power of the protein dataset. This was apparent when all the features of the samples defined as benign hyperplasia and different malignant variables was used for the clustering analysis. We therefore sought to determine whether the subset of 15 proteins 


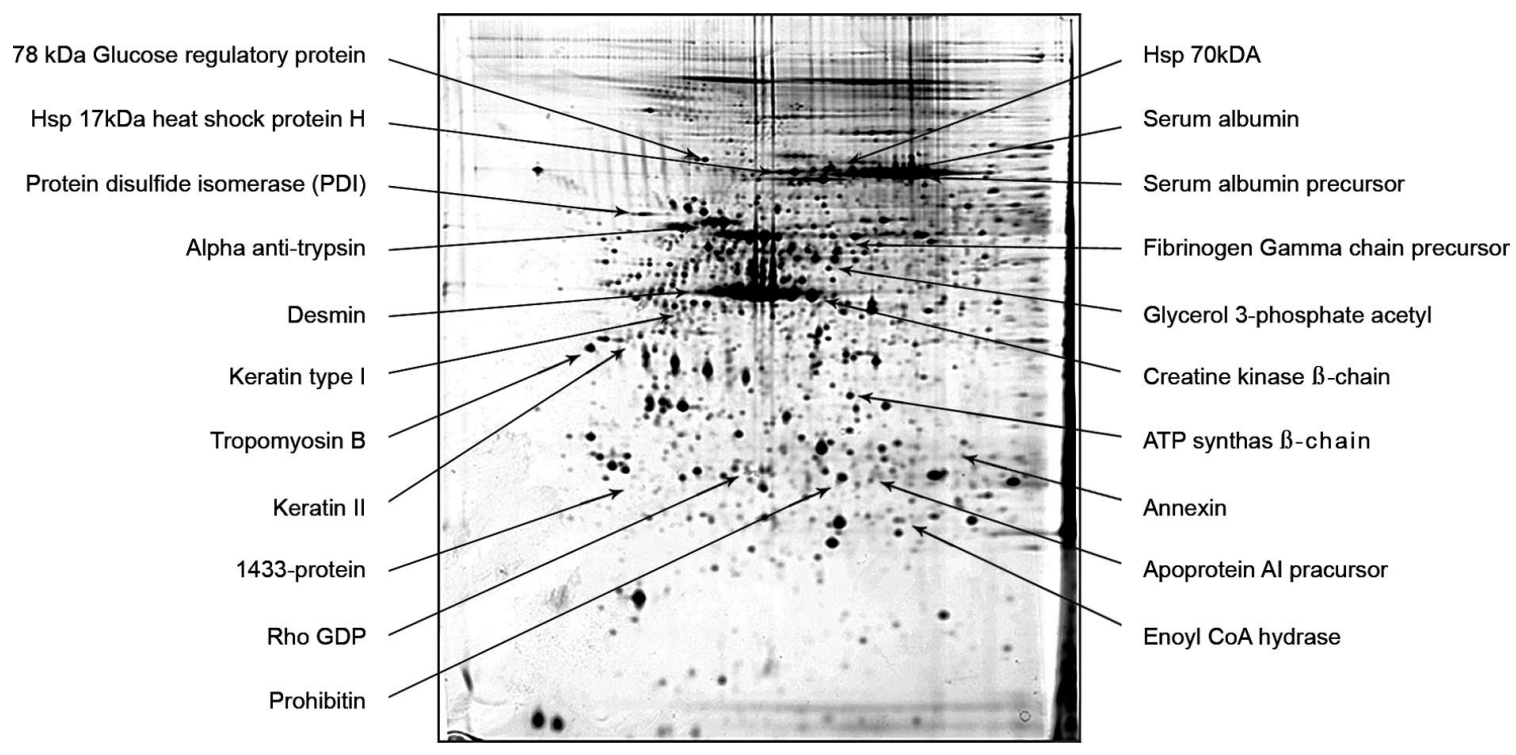

Figure 2. A representative 2-DE gel image of a prostate tumor showing some of the identified differentially expressed proteins between benign hyperplasia and different grades of prostate carcinomas.

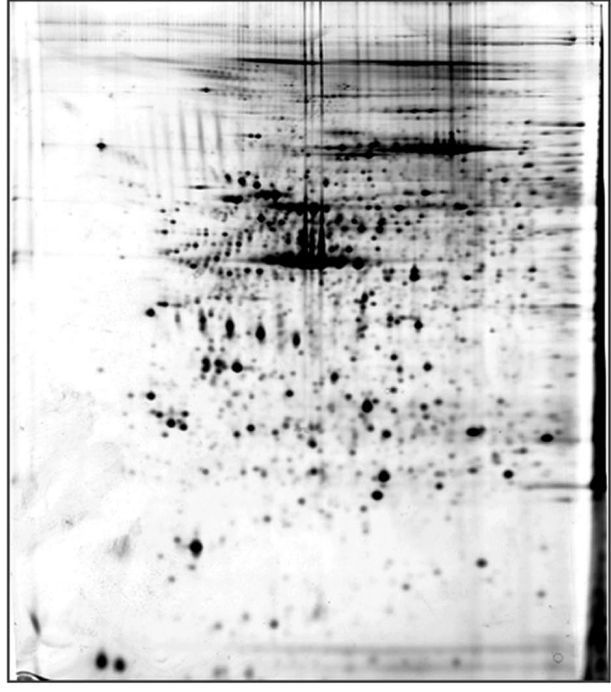

BPH - UP22-H65J-Raw 2-D Image

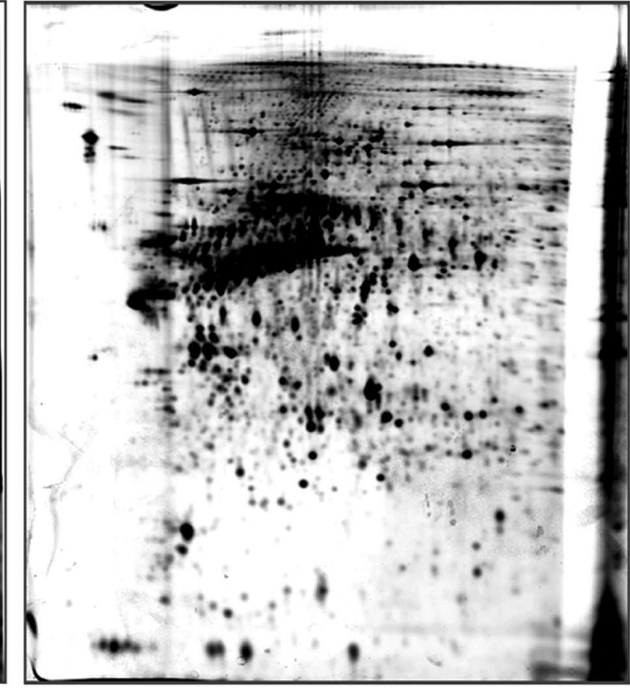

PCa - UP33-H67J-Raw 2-D Image

Figure 3. Representative 2-DE gels derived from one of the prostate hyperplasia and prostate cancer samples.

identified in this study, as well as in previously published studies, could be used for clustering according to Gleason grade classification of malignant prostate cancer samples as described below.

Protein expression in malignant tumors of different grades. A set of eight prostate cancer tissues of different histological Gleason scores were analyzed. Four of the eight samples had Gleason scores of 5, while the remaining four samples had scores of 7. Because of the small sample size, the Gleason 5 samples in this analysis will be referred to as (low malignant) and Gleason 7 as (high malignant tumors) as adapted from the previously described Gleason scoring system (21). Furthermore, the old Gleason scores was applied in this study as against the modified Gleason system reported after the Gleason consensus conference in 2005 (22).

The expression levels of 20 protein spots were significantly differentially expressed between Gleason 5 and 7 samples $(\mathrm{p}<0.05,98 \% \mathrm{CI})$ using Student's t-test. This dataset of 20 protein spots were used in the cluster analysis of malignant tumors with different Gleason grading scores and all samples were correctly classified (Fig. 4A and C). Only eight of the 20 spots were among the 15 identified proteins that have been previously reported to differ in their expression levels between benign and malignant prostate tumors by other laboratories. We then used the expression patterns of the same 15 biomarkers for clustering analysis of malignant tumors of low and high Gleason scores and all samples were correctly classified as 
Table II. Identified differentially expressed proteins between benign prostate hyperplasia and prostate cancer.

\begin{tabular}{|c|c|c|c|c|c|c|c|}
\hline $\begin{array}{l}\text { Gel } \\
\text { SSP no. }\end{array}$ & $\begin{array}{c}\text { Accession } \\
\text { no. }\end{array}$ & Description & MW & $\mathrm{pI}$ & Score & $\begin{array}{c}\text { Probability } \\
(\%)\end{array}$ & $\begin{array}{c}\text { Coverage } \\
(\%)\end{array}$ \\
\hline 1211 & P06468 & Tropomyosin $\beta$ chain fibroblast and epithelial ${ }^{a}$ & 32969 & 4.4357 & 10.4396 & 32.77 & 32.3944 \\
\hline 1226 & P05787 & Keratin type II cytoskeletal 8 Cytokeratin $8 \mathrm{~K}^{\mathrm{a}, \mathrm{b}}$ & 53510 & 5.3399 & 11.5553 & 100 & 59.751 \\
\hline 2101 & P35214 & $14-3-3$ protein $\gamma$ protein kinase $C$ inhibitor $p$ & 28153 & 4.606 & 10.9851 & 56.54 & 47.1545 \\
\hline 2506 & P07237 & Protein disulfide isomerase precursor PDI EC $5^{\mathrm{a}}$ & 57080 & 4.5643 & 11.5552 & 99.99 & 47.4409 \\
\hline 3303 & P08727 & Keratin type I cytoskeletal 19 Cytokeratin $19^{\mathrm{b}}$ & 44079 & 4.8591 & 11.5553 & 100 & 73.25 \\
\hline 3305 & Q25472 & Actin muscle type A2 & 42235 & 4.9501 & 10.1042 & 23.43 & 50.5291 \\
\hline 3424 & P17661 & $\operatorname{Desmin}^{\mathrm{a}, \mathrm{b}}$ & 53372 & 5.0295 & 11.0741 & 61.81 & 50.3198 \\
\hline 3510 & P01009 & $\alpha 1$ antitrypsin precursor $^{\mathrm{a}, \mathrm{b}}$ & 46707 & 5.2405 & 11.5474 & 99.22 & 63.1579 \\
\hline 3529 & P05217 & Tubulin $\beta 2$ chain $^{\mathrm{a}}$ & 49799 & 4.602 & 11.2292 & 72.18 & 44.9438 \\
\hline 3603 & P11021 & $78 \mathrm{kDa}$ glucose regulated protein prec GRP $78^{\mathrm{a}, \mathrm{b}}$ & 72288 & 4.8748 & 11.3436 & 80.92 & 56.1162 \\
\hline 5117 & P52565 & Rho GDP dissociation inhibitor $1^{\mathrm{b}}$ & 23192 & 4.8171 & 11.5198 & 96.52 & 29.902 \\
\hline 6306 & P12277 & Creatine kinase B chain EC $2732 \mathrm{~B} \mathrm{CK}^{\mathrm{a}, \mathrm{b}}$ & 42617 & 5.2167 & 11.5473 & 99.21 & 53.0184 \\
\hline 6535 & P02679 & Fibrinogen $\gamma$ chain precursor ${ }^{\mathrm{b}}$ & 51463 & 5.1561 & 11.5547 & 99.94 & 53.6424 \\
\hline 6613 & P08109 & Heat shock cognate $71 \mathrm{kDa}$ protein ${ }^{\mathrm{a}}$ & 70827 & 5.1998 & 10.5211 & 35.55 & 51.548 \\
\hline 6632 & P38646 & Stress 70 protein mitochondrial precursor $75 \mathrm{kDa}$ & 73733 & 5.8975 & 11.5356 & 98.05 & 20.9131 \\
\hline 6632 & P08107 & Heat shock $70 \mathrm{kDa}$ protein $1 \mathrm{HSP} 701^{\mathrm{a}, \mathrm{b}}$ & 70009 & 5.3187 & 11.3633 & 82.53 & 36.5055 \\
\hline 7005 & P30084 & Enoyl CoA hydratase mitochprecursor EC $4^{\mathrm{a}, \mathrm{b}}$ & 31351 & 8.0728 & 11.5552 & 99.99 & 37.5862 \\
\hline 7101 & P35232 & Prohibitin $^{\mathrm{a}, \mathrm{b}}$ & 29785 & 5.4293 & 11.5269 & 97.2 & 25.3676 \\
\hline 7110 & P02647 & Apolipoprotein A I precursor Apo $\mathrm{AI}^{\mathrm{b}}$ & 30758 & 5.4309 & 11.5519 & 99.66 & 67.4157 \\
\hline 7212 & P10719 & ATP synthase $\beta$ chain mitochondrial precursor ${ }^{\mathrm{a}, \mathrm{b}}$ & 56318 & 5.0198 & 10.819 & 47.89 & 45.7467 \\
\hline 7304 & O75643 & Small nuclear ribonucleoprotein $200 \mathrm{kDa}$ helicas & 194354 & 6.2509 & 11.0926 & 62.96 & 23.5744 \\
\hline 7403 & Q9HXW7 & Glycerol 3 phosphate acyltransferase EC 23115 & 94757 & 9.5317 & 11.0348 & 59.42 & 23.2614 \\
\hline 7521 & P05783 & Keratin type I cytoskeletal 18 Cytokeratin $18^{\mathrm{a}, \mathrm{b}}$ & 47897 & 5.1674 & 11.5553 & 100 & 43.8228 \\
\hline 7623 & P02768 & Serum albumin precursor & 69321 & 5.8601 & 11.5553 & 100 & 47.7833 \\
\hline 8106 & P09525 & Annexin A4 Lipocortin IV Endonexin ${ }^{\mathrm{a}, \mathrm{b}}$ & 35729 & 5.7268 & 11.5406 & 98.54 & 43.0818 \\
\hline 8603 & P02768 & Serum albumin precursor ${ }^{\mathrm{b}}$ & 69321 & 5.8601 & 11.5553 & 100 & 49.2611 \\
\hline 3622 & P10809 & $60 \mathrm{kDa}$ heat shock protein mitochondrial precursor ${ }^{\mathrm{b}}$ & 61016 & 5.5503 & 10.7736 & 45.76 & 61.4311 \\
\hline
\end{tabular}

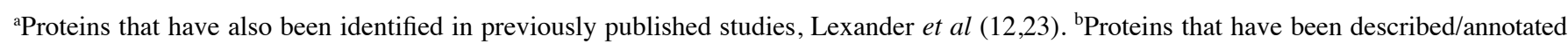
with functional links to different signaling pathways in Ingenuity Pathway Analysis (IPA) database as described in Table III and Fig. 6). ${ }^{\mathrm{c} P M F}$ analyzed 2-3 times at different runs and only concurrent results in all the runs are considered as unambiguous protein identification.

shown in Fig. 4B. The differential expression changes of the subset of 15-biomarker proteins based on Gleason scores is shown in Fig. 5.

Similarities in the proteome of prostate tumors of different ethnic groups. We compared our results with previously published studies on prostate tumors (11-13) and to our knowledge; these samples were obtained from Swedish patients, while samples analyzed in this study were primarily from Saudi men diagnosed with prostate tumor.

We observed a high degree of similarity between our generated 2-DE maps and some of the previously published 2-DE images of prostate tumor tissues. Although we did not have access to high-resolution digital copies of the published gel images, we used computer-assisted image analysis for the comparisons. Despite this caveat, using the low-resolution image of one of the published 2-DE-gels, we successfully matched $>50 \%$ of the gel spots from our gel with the published image (data not shown).

In this study, we used IPG strips of similar $\mathrm{pH}$ gradient (4.0-7.0 non-linear) for the first dimension iso-electric focusing as in other reported studies. However, our 2nd dimension SDS-polyacrylamide gels consist of $12 \%$ homogeneous gels as opposed to the linear gradient (10-13\%) gels that were used in the others studies $(12,23,24)$ and our previously published data $(10,11)$. Representative 2-DE gels derived from one of the prostate hyperplasia and prostate cancer samples are shown in Fig. 3. Apart from a high degree of similarity in the gel images derived from these sample cohorts, we also observed similar expression changes between different tumor groups and different malignant grades. More than 60\% (15/22) of the identified differentially expressed proteins between BPH and prostate cancers in this present study have also been previously reported (Table II and Fig. 2). Interestingly, a similar observation 

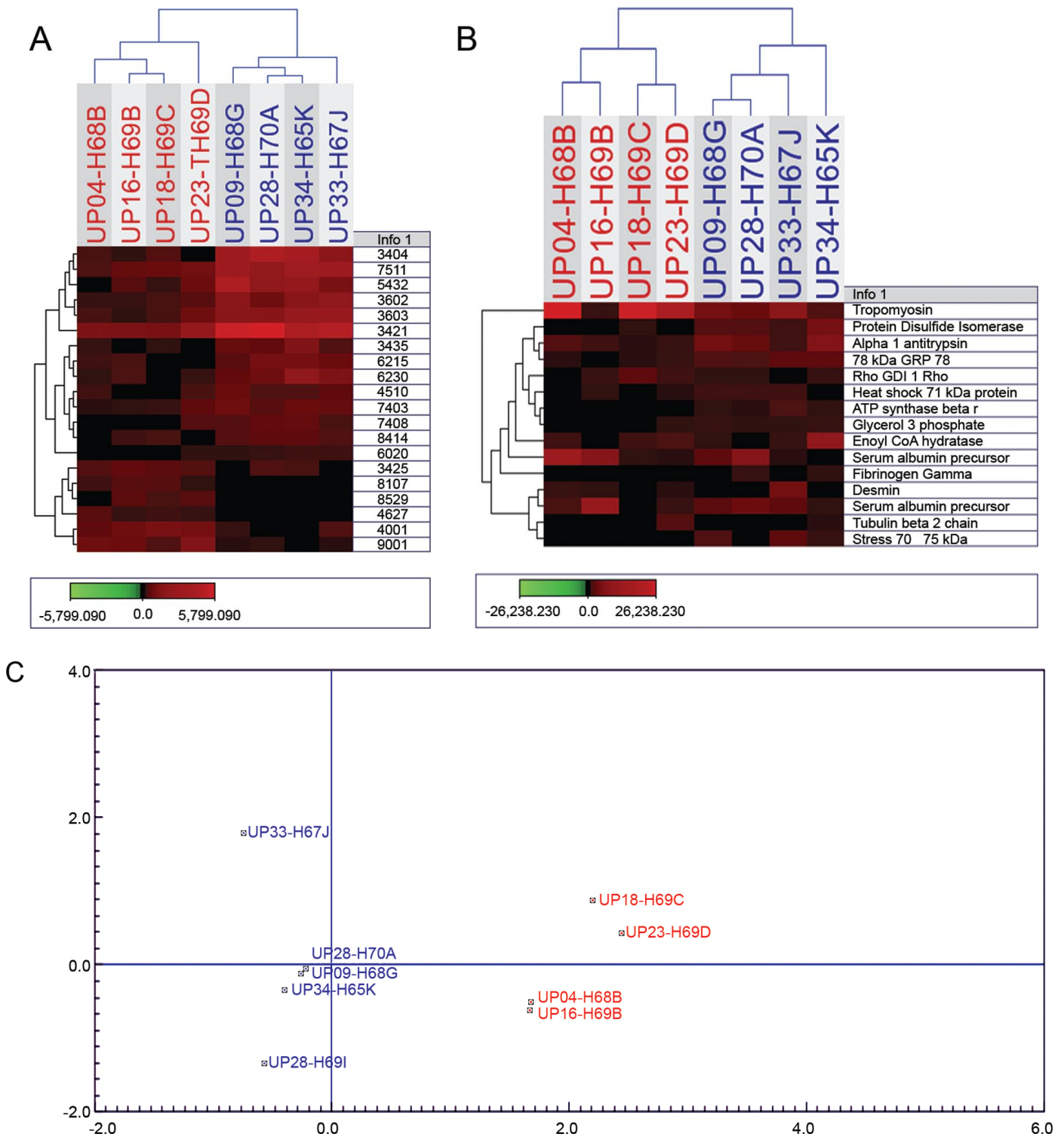

Figure 4. (A) Hierarchical Cluster analysis using the expression patterns of 20 proteins that are differentially expressed between Gleason 5 (low grade) and Gleason 7 (high grade) malignant prostate tumors. (B) Hierarchical Cluster analysis using the expression patterns of 15 proteins that have been identified both in this study and in previously published studies. The names of several of the identified proteins are indicated in the dendrogram (red, high grade Gleason 7; and blue, low-grade Gleason 5 cancers). The dendrogram was generated using the Bray Curtis distance metric and an average linkage clustering method from the J-Express software. (C) The correspondence analysis of the same dataset.

was found among identified proteins in this study between malignant tumors of different stage and Gleason scores and those reported by other studies. These results indicate; that inter laboratory comparison of 2-DE maps is possible and that 2-DE findings are reproducible with adequate experimental settings. Therefore, the proteins identified in this study are considered as potential markers for prostate cancer and BPH.

\section{Discussion}

Several studies have described marked alterations in prostate tissue and serum samples from prostate cancer patients using methods including immunohistochemistry, tissue micro-arrays and SELDI protein-chips (25-27). However, very few studies have analyzed human prostate tissues using 2-DE based protein expression analysis. It is necessary that potential protein markers, identified by proteome analysis, be verified by other methods such as immunohistochemistry using large numbers of representative tumor materials. The obvious limitation of such validation studies is lack of commercially available antibodies to most of the described proteins. An alternate form of validation should be considered, if independent investigators, using similar disease samples can reproducibly obtain similar results.

This discussion will focus on the reproducibility of published 2-DE data on prostate tumors, and the use of data derived from this study as a validation and as a proof of concept for the discovery of biomarkers for prostate tumors.

Studies of breast, ovarian and prostate tumors have shown that 2-DE gels are very reproducible when protocols are carefully developed $(12,15,16,20)$. In this present study, analyzed samples were procured by either TURP (all BPH) or radical prostatectomy (all prostate cancer samples) and immediately processed for 2-DE as previously described 


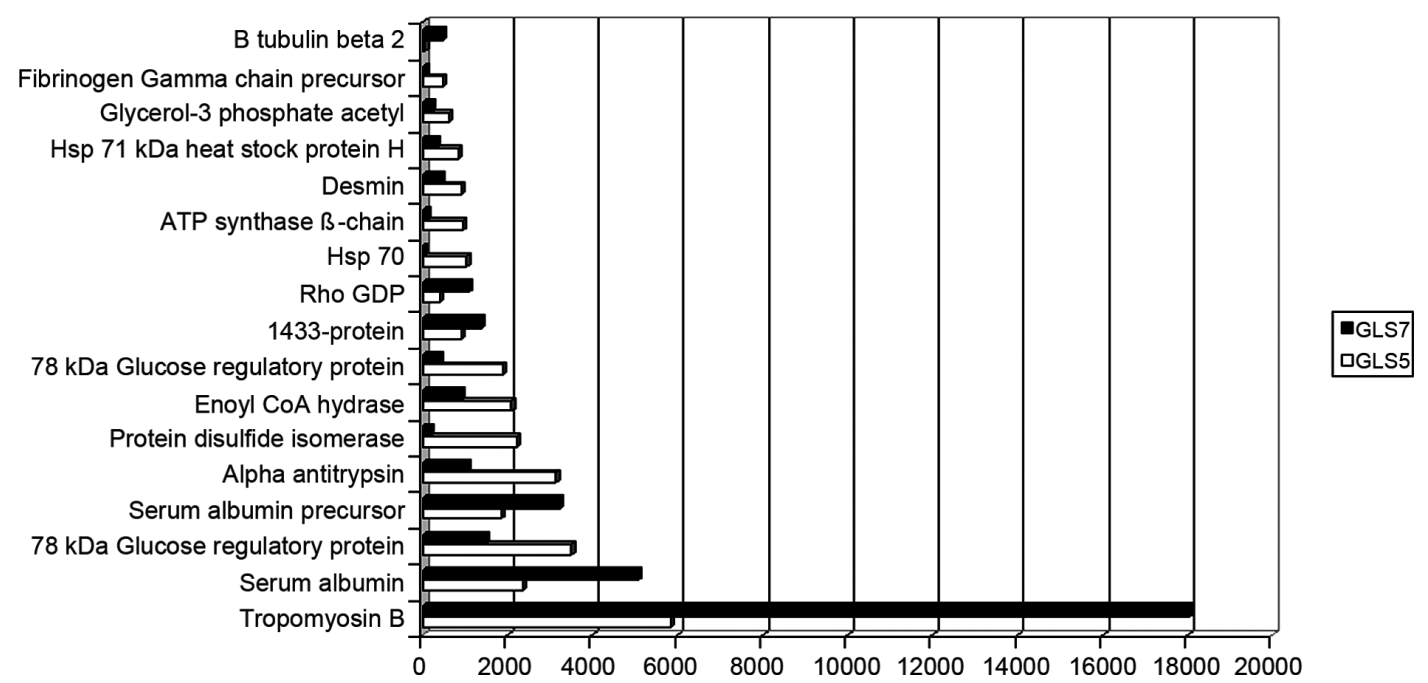

Figure 5. Bar chart of the differentially expressed proteins between Gleason scores 5 and 7 (black-filled, Gleason 7; and white-empty, Gleason 5 tumors).

$(10,11,15)$. Even though the BPH samples were taken from the central zone of the prostate since they are procured by TURP, while the prostate cancer samples are typically derived from the peripheral region of the prostate gland, the resolutions of total separated proteins spots from our two sample procurement methods were very similar. Differences in protein expression patterns between benign and malignant tumors could be attributed to either true biological changes or changes due to differences in sample collection methods as previously reported (23). The latter possibility was seriously considered; therefore, the focus of the analysis in this study is directed at changes in protein expression between malignant tumors of different grades that were procured by same collection method (radical prostatectomy).

We observed changes in the expression of 47 proteins between BPH and prostate carcinomas samples. The expression levels of these protein spots allowed discrimination between benign hyperplasia and prostate carcinomas (data not shown). Previously published results, using fresh prostate tumor samples $(10-12,24)$, reported similar findings to that of this study and also highlighted the possibility of disease classification using an artificial learning model of multiple polypeptides $(13,18,19)$. Fifteen of the $22(68 \%)$ proteins identified in this study have been previously described to differ in expression between benign hyperplasia and prostate carcinomas $(12,13,23,24)$. This observation validates previously reported protein changes in prostate tumors $(12,13,23,24)$. The fact that these datasets can be largely reproduced at different laboratories is encouraging.

Tumor staging and Gleason grading system remains one of the most powerful prognostic predictors in prostate cancer $(28,29)$. However, the use of these parameters in combination with measurement of serum PSA pre- and post-radical prostatectomy can not conclusively predict recurrence or progression of the disease (30). This has drawn caution on the prognostic evaluation of prostate tumors procured by radical prostatectomy and led to a call for the discovery of more objective quantitative biomarkers. The 'low malignantGleason 5 cancers' showed $>2$-fold differences in the expression of $78 \mathrm{kDa}$ glucose regulatory protein, $\alpha$ antitrypsin, protein disulfide isomerase, enoyl CoA-hydrase, Hsp-70, ATP synthase $\beta$-chain, desmin, Hsp-71 kDa, glycerol-3 phosphate acetyl, and fibrinogen $\gamma$ chain precursor than the 'high malignant cancers with a Gleason score of 7'. In addition, these Gleason 5 cancers showed $<2$-fold decrease in the level of expression of Rho-GDP, tropomyosin-B and Tubulin- $\beta$ - 2 . Even though the sample size in this study is small, our findings are similar to, and support, those described in other studies with larger sample sizes (13). Furthermore, 12 of 23 proteins identified in another previous study that differs significantly between BPH and prostate cancer are among the 15 proteins validated in this study, although their usefulness for discriminating tumors of different Gleason grades was not evaluated (13).

The results of this study can offer alternative complementary approach to the classical Gleason scoring system. The Gleason grading system from tissue samples can be mirrored into proteomics quantitative data for more objective assessment of disease status. The potential of using multiple markers for disease classification against the use of single markers has been advocated. The expression levels of 190 protein spots from 2-DE gels were able to discriminate between ovarian tumors of low malignant potential from ovarian carcinomas (19). Similarly, we are among the first to discriminate BPH from carcinoma based on the expression levels of only nine proteins $(10,13)$. As shown in Fig. 4, the expression levels of 20 protein spots was able to discriminate between G5 and G7 prostate tumors using unsupervised hierarchical cluster analysis. This implies that it will be difficult to define a universal protein panel for BPH and prostate carcinoma. Therefore, specific marker sets need to be defined for different malignant tumor sub-types.

The majority of molecules identified in this study are involved in various signaling pathways and mostly regulates among others, proliferation, survival, cell cycle progression and apoptosis and these molecules are located mostly in the cytoplasm and only a few are located in the nucleus and extracellular space. While some of these molecules act as transporters, others act as enzymes and transcription regulators as presented in Table III and Fig. 6. 


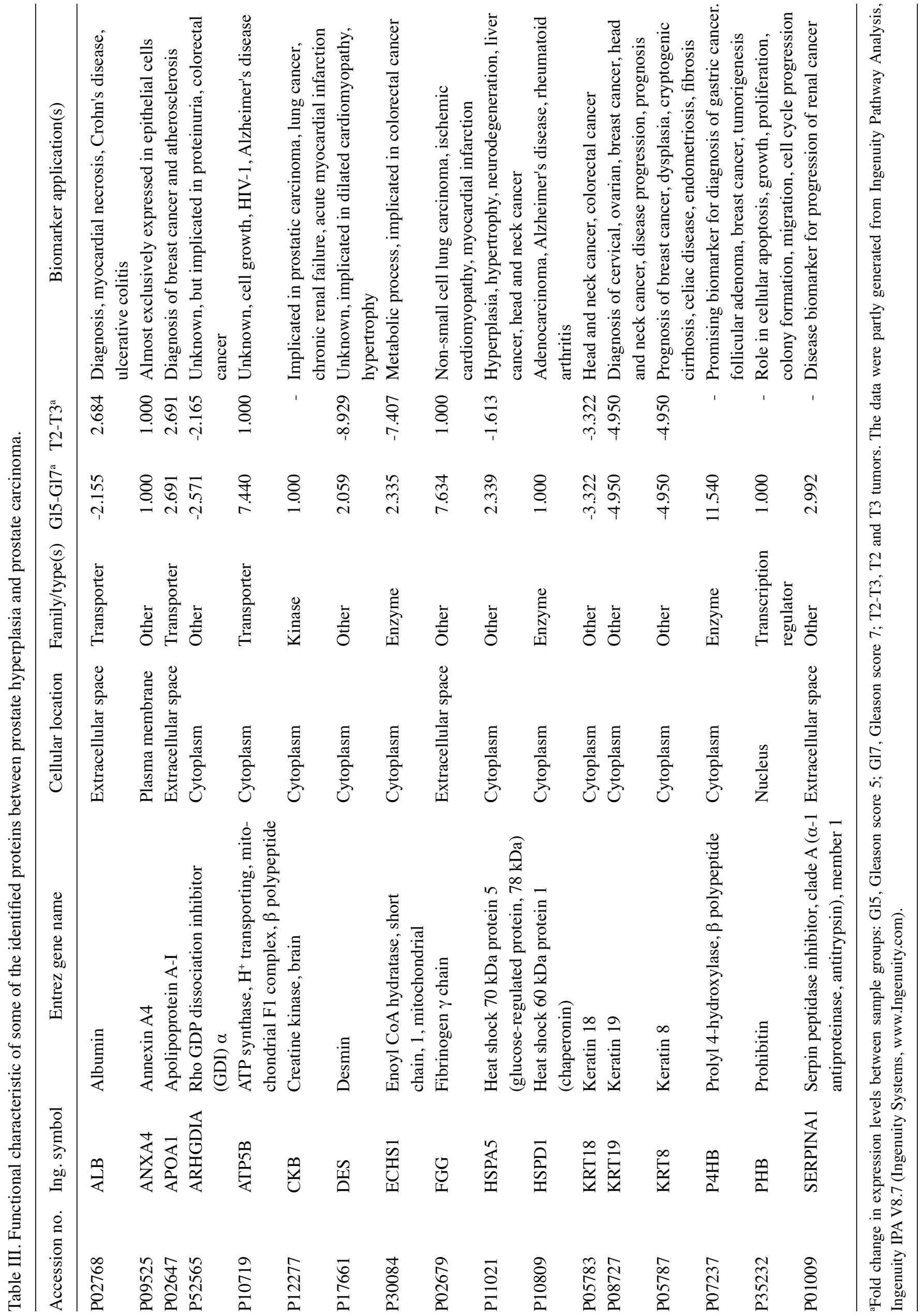




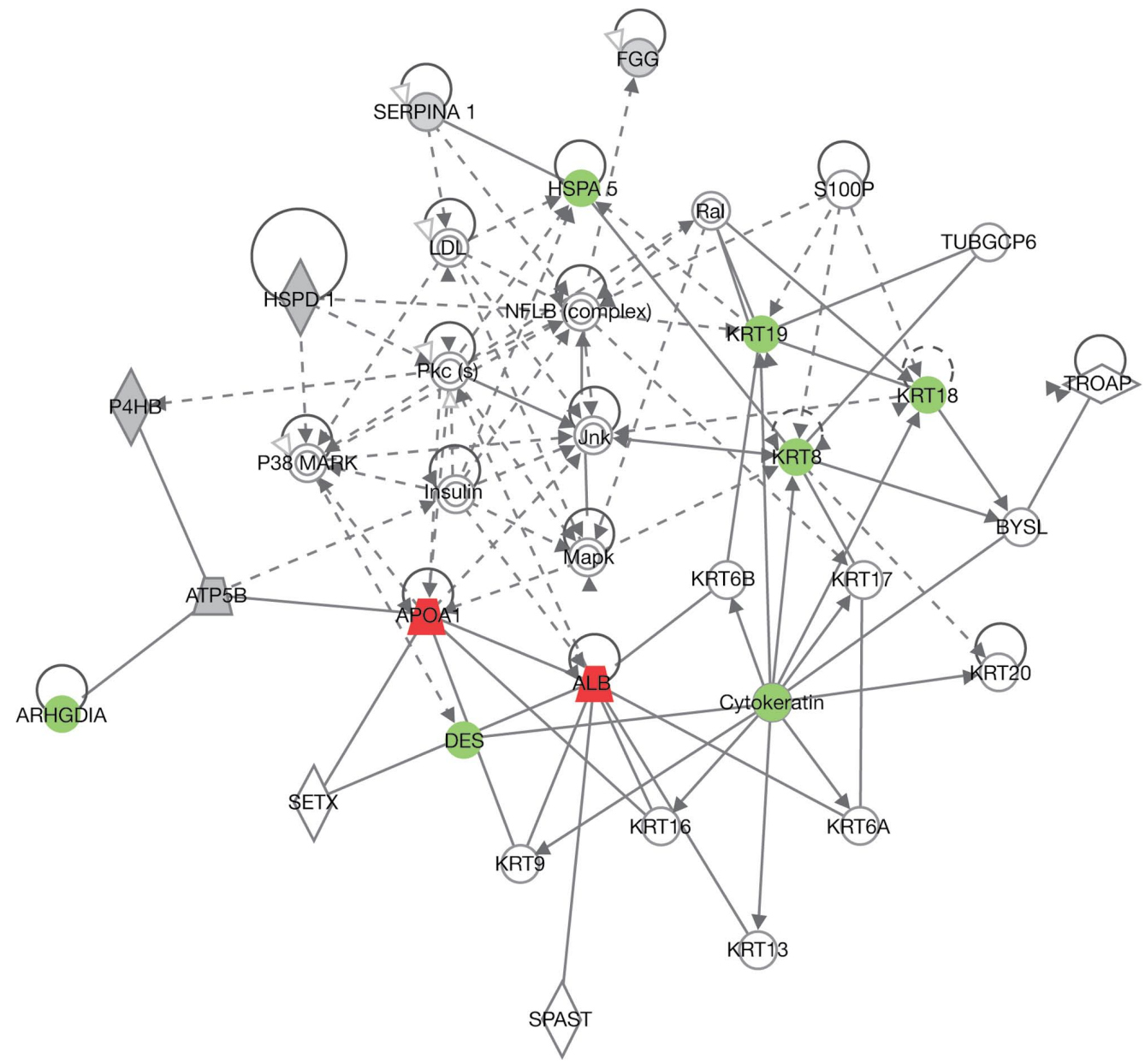

Figure 6. Canonical pathway analysis of network signaling of identified proteins in prostate cancer. Some of the identified proteins were mapped in Ingenuity database and represented in multiple sub-signaling networks and mostly regulates among others: proliferation, survival, cell cycle progression and apoptosis and these molecules are located mostly in the cytoplasm and only a few are located in the nucleus and extracellular space. While some of these molecules act as transporters, others act as enzymes and transcription regulators. The connection and the expression profile of some of the proteins are as indicated. Red indicates an up-regulated protein, and green color is indicative of down-regulation, a direct connection is by solid line and broken lines indicates an indirect interaction between different molecules ALB, albumin; APOA1, apolipoprotein A-I; ARHGDIA, Rho GDP dissociation inhibitor (GDI) $\alpha$; DES, desmin; HSPA5, heat shock 70-kDa protein 5 (glucose-regulated protein, 78 kDa); KRT18, Keratin 18; KRT19, Keratin 19; KRT8, Keratin 8. The network analysis was done in Ingenuity Pathway Analysis program (IPA V8.7).

The limitation of this study is the small sample size however; the reproducibility of the results in line with previously published data on prostate cancer is very encouraging. The majority of analyzed samples in this study have had a follow-up of approximately 2-3 years and all patients are alive as at the time of this study. It is anticipated that in the near future, the prognostic evaluation based on using multivariate analysis of these proteins will be further validated when other clinical parameters such as residual free survival and patient outcome becomes available.

Previous studies have used tumors from Swedish patients (personal communication), while all samples reported in this study were obtained from Saudi men diagnosed with prostate tumors. The prevalence of prostate cancer is higher in Sweden than in Saudi Arabia. For example, the 1 year prevalence rates for Sweden and Saudi Arabia are 7346 and 377, while the 5-year prevalence rates are 28082 and 1236 respectively (31). It is interesting that the differences in protein expression during malignant progression are so similar in tumors from patients with different ethnical origin and with different disease prevalence. This study is the first attempt to characterize protein expressions in prostate tumors from patients of diverse ethnic backgrounds.

This study is the first of its kind to validate panel of proteins for objective classification and potential prognostic monitoring of prostate adenocarcinoma. These markers are found in prostate tumors in populations that differ with regard to the prevalence of prostate cancer (i.e., high in Sweden, low in Saudi Arabia); indicating that $\mathrm{BPH}$ and prostate cancers are biologically 'homogeneous' in their protein expression patterns between different ethnic populations.

These validated marker sets could be useful for accurate diagnosis of prostate cancer and prognostic monitoring. However, further characterization and validation on clinical significance of the identified potential protein markers on larger sample size of prostate adenocarcinoma is warranted.

\section{Acknowledgements}

The authors wish to acknowledge the support of Dr Sultan Al Sedairy and the Research Center Administration at the King Faisal Specialist Hospital \& Research Center. We are thankful 
for the support and logistic assistance from Mr. Hakem Al Enazi and Logistics and Facilities Management Office. This work was supported by grants from the King Faisal Specialist Hospital and Research Centre (RAC Project no. 2050 026). We thankfully acknowledge Professor Stig Linder for his help in critical review of this report.

\section{References}

1. Ziada AM and Crawford ED: Advanced prostate cancer. Prostate Cancer Prostatic Dis 2: 21-26, 1999.

2. Jemal A, Siegel R, Ward E, Hao Y, Xu J and Thun MJ: Cancer statistics, 2009. CA Cancer J Clin 59: 225-249, 2009.

3. Socialstyrelsen: Statistics - Health and Diseases Cancer Incidence in Sweden 2007, www.socialstyrelsen.se. Journal 2007.

4. Abdalla I, Ray P and Vijayakumar S: Race and serum prostatespecific antigen levels: current status and future directions. Semin Urol Oncol 16: 207-213, 1998.

5. Dorkin TJ and Neal DE: Basic science aspects of prostate cancer. Semin Cancer Biol 8: 21-27, 1997.

6. Greene KL, Albertsen PC, Babaian RJ, et al: Prostate specific antigen best practice statement: 2009 update. J Urol 182 2232-2241, 2009.

7. Charrier JP, Tournel C, Michel S, Dalbon P and Jolivet M: Two-dimensional electrophoresis of prostate-specific antigen in sera of men with prostate cancer or benign prostate hyperplasia. Electrophoresis 20: 1075-1081, 1999.

8. Torti FM: Genitourinary system. Curr Opin Oncol 9: B75-B77, 1997.

9. Lalani EN, Stubbs A and Stamp GW: Prostate cancer; the interface between pathology and basic scientific research. Semin Cancer Biol 8: 53-59, 1997.

10. Alaiya A, Roblick U, Egevad L, et al: Polypeptide expression in prostate hyperplasia and prostate adenocarcinoma. Anal Cell Pathol 21: 1-9, 2000

11. Alaiya AA, Oppermann M, Langridge $\mathrm{J}$, et al: Identification of proteins in human prostate tumor material by two-dimensional gel electrophoresis and mass spectrometry. Cell Mol Life Sci 58: 307-311, 2001

12. Lexander H, Hellman U, Palmberg C, et al: Evaluation of two sample preparation methods for prostate proteome analysis. Proteomics 6: 3918-3925, 2006.

13. Lexander H, Palmberg C, Hellman U, et al: Correlation of protein expression, Gleason score and DNA ploidy in prostate cancer. Proteomics 6: 4370-4380, 2006.

14. Alaiya AA, Franzen B, Fujioka K, et al: Phenotypic analysis of ovarian carcinoma: polypeptide expression in benign, borderline and malignant tumors. Int J Cancer 73: 678-683, 1997.
15. Alaiya AA, Franzen B, Moberger B, Silfversward C, Linder S and Auer G: Two-dimensional gel analysis of protein expression in ovarian tumors shows a low degree of intratumoral heterogeneity. Electrophoresis 20: 1039-1046, 1999.

16. Franzen B, Auer G, Alaiya AA, et al: Assessment of homogeneity in polypeptide expression in breast carcinomas shows widely variable expression in highly malignant tumors. Int J Cancer 69: 408-414, 1996.

17. Alaiya A, Al-Mohanna M and Linder S: Clinical cancer proteomics: promises and pitfalls. J Proteome Res 4: 1213-1222, 2005.

18. Alaiya AA, Franzen B, Hagman A, et al: Molecular classification of borderline ovarian tumors using hierarchical cluster analysis of protein expression profiles. Int J Cancer 98: 895-899, 2002.

19. Alaiya AA, Franzen B, Hagman A, et al: Classification of human ovarian tumors using multivariate data analysis of polypeptide expression patterns. Int J Cancer 86: 731-736, 2000.

20. Franzen B, Hirano T, Okuzawa K, et al: Sample preparation of human tumors prior to two-dimensional electrophoresis of proteins. Electrophoresis 16: 1087-1089, 1995.

21. Gleason DF and Mellinger GT: Prediction of prognosis for prostatic adenocarcinoma by combined histological grading and clinical staging. J Urol 111: 58-64, 1974.

22. Epstein JI: An update of the Gleason grading system. J Urol 183: 433-440, 2010.

23. Lexander H, Franzen B, Hirschberg D, et al: Differential protein expression in anatomical zones of the prostate. Proteomics 5: 2570-2576, 2005.

24. Lexander H, Palmberg C, Auer G, et al: Proteomic analysis of protein expression in prostate cancer. Anal Quant Cytol Histol 27: $263-272,2005$

25. Cordon-Cardo C, Koff A, Drobnjak M, et al: Distinct altered patterns of $\mathrm{p} 27^{\mathrm{KIPl}}$ gene expression in benign prostatic hyperplasia and prostatic carcinoma. J Natl Cancer Inst 90: 1284-1291, 1998.

26. Gannon PO, Koumakpayi IH, Le Page C, Karakiewicz PI, Mes-Masson AM and Saad F: Ebp1 expression in benign and malignant prostate. Cancer Cell Int 8: 18, 2008.

27. Liu AY, Zhang H, Sorensen CM and Diamond DL: Analysis of prostate cancer by proteomics using tissue specimens. J Urol 173: 73-78, 2005 .

28. Epstein JI: What's new in prostate cancer disease assessment in 2006? Curr Opin Urol 16: 146-151, 2006.

29. Epstein JI, Allsbrook WC Jr, Amin MB and Egevad LL: Update on the Gleason grading system for prostate cancer: results of an international consensus conference of urologic pathologists. Adv Anat Pathol 13: 57-59, 2006.

30. Sakr WA and Grignon DJ: Prostate. Practice parameters, pathologic staging, and handling radical prostatectomy specimens. Urol Clin North Am 26: 453-463, 1999.

31. Globocan-2002: The Globocan 2002 database at http://www-dep. iarc.fr/globocan/database.htm. 2002. 